\title{
AN OPTICAL METHOD FOR MEASURING TEMPERATURE DISTRIBUTION AND CONVECTIVE HEAT TRANSFER
}

\author{
By R. B. Kennard
}

ABSTRACT

In order to avoid the errors inherent in material thermometers, a method has been devised whereby the temperatures in the region of heated air near a hot surface may be determined from the displacement of the fringes in an interferometer.

Photographs were taken of the interference fringes near a hot vertical plate and near horizontal heated cylinders. The temperature distribution and the convective heat transfer were determined from measurements made on enlargements of these photographs. It is shown that the temperature distribution does not correspond to that called for by the film theory.

\section{CONTENTS}

I. Introduction

II. Methods and procedure... 789

III. Calculation of results

1. General........ 790

2. End correction

3. Effects of aberration

IV. Vertical hot plate

1. Experimental results

2. Discussion of Figure 6

3. Calculation of convective heat transfer.... 797

V. Horizontal heated cylinders

1. Experimental results.... 798

2. Discussion _........ 802

VI. Comparison with film theory

1. Vertical plate

2. Horizontal cylinders

VII. Conclusion .......

VIII. Acknowledgment .

Note on Hansen paper... 805

\section{INTRODUCTION}

The exchange of heat between the surface of a solid and a fluid in contact with it is one of the most important types of heat transfer. A complete mathematical solution of the problems involved must combine the hydrodynamic laws for the motion of a viscous fluid in both laminar and turbulent flow, with Fourier's laws of heat conduction. Because of the extreme complexity of the problem no complete solution has as yet been made. Many theoretical workers have sought approximate solutions by making arbitrary assumptions. To avoid the mathematical difficulties involved in the analysis of turbulent flow, the first approximation is to consider the problem as involving laminar flow only. The combination of the laws of lami- 
nar flow and heat conduction is further simplified by assuming a nonviscous fluid. This method was followed by Boussinesq ${ }^{1}$ and Russell. $^{2}$ The calculated results, however, differ widely from those observed experimentally, and the method is of little value in the direct calculation of heat transfer.

The method of dimensional analysis ${ }^{3456}$ has been of great value in extending the use of experimental data on convective heat transfer, but does not indicate what takes place in the boundary region between the solid and the fluid.

In calculating the heat transferred by convection, it is generally assumed that the heat is transferred by conduction through a thin film of fluid in contact with the solid. On one side of this film the temperature is that of the surface of the solid, and on the other side that of the fluid at a great distance. The thickness of such a film may be calculated from measurements of $(a)$ the total heat transferred by convection, $(b)$ the total temperature drop, and $(c)$ the average thermal conductivity of the fluid. The conducting film as defined in this manner is purely hypothetical. However, since the thickness of the film is in fact not known, it is the practice to combine the thickness and thermal conductivity into the film coefficient ${ }^{7} h$

$$
h=\frac{K}{L}
$$

where $K$ is the conductivity and $L$ the film thickness. These film coefficients, or coefficients of heat transfer, determined experimentally over a wide variety of conditions, can be adapted to particular problems by the methods of dimensional analysis.

The film hypothesis was placed on a theoretical basis by Langmuir. ${ }^{8}$ He assumed that near the surface the flow would be laminar, with the velocity increasing from zero at the surface to a maximum critical velocity. When the critical velocity was exceeded, the flow would break into turbulent motion. Langmuir assumed that because of this turbulence the temperature throughout the turbulent region would be essentially the same as that of the ambient fluid at a great distance. In other words, this theory would lead to the conclusion that the temperature drop would be confined to the region of laminar flow. Reasoning from the kinetic theory of gases, and taking into account the change of viscosity and heat conductivity with temperature, Langmuir deduced certain laws for the thickness of this film. From these laws it was possible to correlate the observations of many observers on convective heat transfer for wires and cylinders in different gases. The correlation was remarkably good over a wide range of pressures and temperatures of the gas, and of cylinder diameters.

In order to determine how closely the film theories agree with the physical facts, it is necessary to measure the temperature and the

1 Boussinesq, Comptes Rendus, vol. 132, p. 1383, 1901.

2 Alex. Russell, Phil. Mag., vol. 20, p. 591, 1910.

3 Buckingham, Phys. Rev., vol. 4, p. 345, 1914.

- Davis, Phil. Mag., vol. 40, p. 692, 1920; vol. 41, p. 899, 1921.

S Rice, Trans. A. I. E. E., vol. 42, p. 653, 1923.

- Fishenden and Saunders, Engineering, vol. 130, p. 177, 1930.

7 Walker, Lewis, and McAdams, Principles of Chemical Engineering (2d ed.), pp. 134-154.

8 Langmuir, Trans. A. I. E. E., vol. 31, pt. 1, p. 1229, 1912.

- Langmuir, Trans. A. I. E. E., vol. 32, p. 301, 1913. 
velocity distribution in the fluid near the hot surface. Griffith and Davis ${ }^{10}$ made such an investigation for free convection in air in front of a vertical hot plate. Jürges, ${ }^{11}$ Ludowici, ${ }^{12}$ and Elias ${ }^{13}$ made a similar study for forced convection. In these investigations, the air velocities were measured by a hot wire anemometer, and the temperatures either by the resistance of a fine platinum wire ${ }^{10}$ or by thermocouples..$^{11} 1213$

There are inherent objections to the introduction of any material thermometers under these conditions. The effects of radiation and the conduction of heat along the leads may cause the temperature of the thermometer to differ materially from that of the air surrounding it. Furthermore, the introduction of any stationary solid into a moving fluid will disturb the conditions of temperature and flow. In the case of small wires this disturbance decreases only very slowly with the diameter, for, at the surface of the wire, regardless of its size, the velocity of the fluid is reduced to zero. There is therefore a

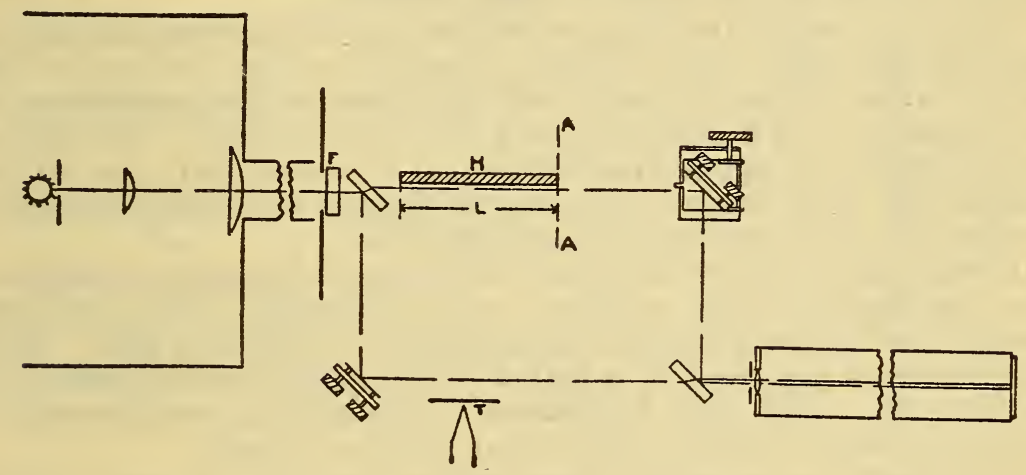

FiguRE 1.-Experimental arrangement

$H$ is the heated plate or cylinder of length $L . A A$ is the plane in which the fringes were brought to a virtual focus. $F$ is a filter which transmits only the green mercury light. $T$ is a shielded thermal couple

limit to the advantage gained by using extremely fine wires as resistance thermometers or as thermocouples.

\section{METHODS AND PROCEDURE}

In order to avoid the errors incident to the use of material thermometric devices the following method has been devised whereby the temperature distribution in the air near a heated surface may be determined by the displacement of the fringes in an interferometer.

The experiments were performed in an inside basement room, the walls and ceiling of which were lined with 4 inches of cork. There were, therefore, no complications caused by vibration, or by stray convection currents set up by unequal temperatures within the room.

In Figure 1 is shown a plan view of the interferometer together with the light source, the heated surface, and the camera.

10 Griffith and Davis, Food Investig. Board, No. 9, 1922.

11 Jürges, Gesundheits Inginewr, vol. 19, p. 5, 1924.

13 Ludowici, Z. d. V. D. I., vol. 70, p. 1122, 1926.

18 Elias, Z. f. Ang. Math. u Mech., vol. 9, p. 434, 1929. Elias, Z. 1. Ang. Math. u Mech., vol. 10, p. 1, 1930. 
The light from a mercury lamp passing through a $10 \mathrm{~mm}$ aperture is brought to a slightly convergent beam by two condensing lenses. The use of two lenses is found convenient as the focal length of the combination can be varied by moving the inner lens, and in this way the degree of convergence of the light beam can be controlled without moving the lamp or the large outer lens. The lamp and lenses are housed in a light-tight cabinet separated from the interferometer by a $60 \mathrm{~cm}$ tube. This was done in order to reduce the effect of stray air currents caused by the heat of the lamp. At the end of the tube is a shutter so that the light of the lamp can be cut off completely. Before entering the interferometer the light passes through a Wratten 77 filter $F$, which will transmit only the mercury green light.

A Zehnder interferometer was chosen, as the path of light goes past the hot surface only once, as compared to twice for the Michelson instrument. For the same temperature rise and the same fringe shift the width of the heated surface could be made twice as great and the end corrections proportionally reduced.

The heated body $H$ was placed in one arm of the interferometer as shown in the figure. The set was adjusted so that the axis of the beam of light accurately coincided with that of the interferometer and passed by the hot surface with grazing incidence. The fringes were brought to a virtual focus at the end of the hot body nearest the camera (line $A A$ ), except in the case of the hot wire when they were brought to a focus at the middle of the wire.

In order to avoid any distortion of the interferometer by radiation from the heated body, water at room temperature was syphoned through the hollow frame which is made of 2 -inch iron pipe. This frame rests on dry sand in a hollow square supported 34 inches off the floor by a rigid open framework. This gives a free flow of air about the heated surface without any chimney effects.

The camera has a length from lens to plate of $136 \mathrm{~cm}$. A 21/2diopter spectacle lens is used for the objective, giving a magnification of 2.4 diameters. Enlarged positives were made from the negatives, the total magnification being approximately 7 diameters.

In taking all photographs a double exposure was made on the same plate to show a millimeter scale in the object field. The temperature distribution was determined from the displacement of the fringes and the dimensions as obtained from the photograph of the scale.

Figures $5,7,9(A), 9(B)$, and 11 show the type of photographs of interference fringes that may be taken with this instrument.

\section{CALCULATION OF RESULTS}

\section{GENERAL}

Let $A$ and $B$ (fig. 2) be two adjacent interference fringes formed by monochromatic light passing through the column of air adjacent to the heated surface $H$. If the temperature were uniform throughout, the center line of fringe $A$ instead of the center line of fringe $B$ would pass through the point $P$. The shift of one fringe at the point $P$ indicates that there is one less wave of light in the path through $P$ than there would be if the temperature at $P$ were the same as at $Q$. 
The temperature of the air is obtained from its density which is related to the refractive index by the Lorenz-Lorentz equation ${ }^{14}$

$$
\frac{n^{2}-1}{n^{2}+2}=C \rho
$$

where $n$ is the index of refraction, $\rho$ the density, and $C$ is a constant characteristic of the fluid.

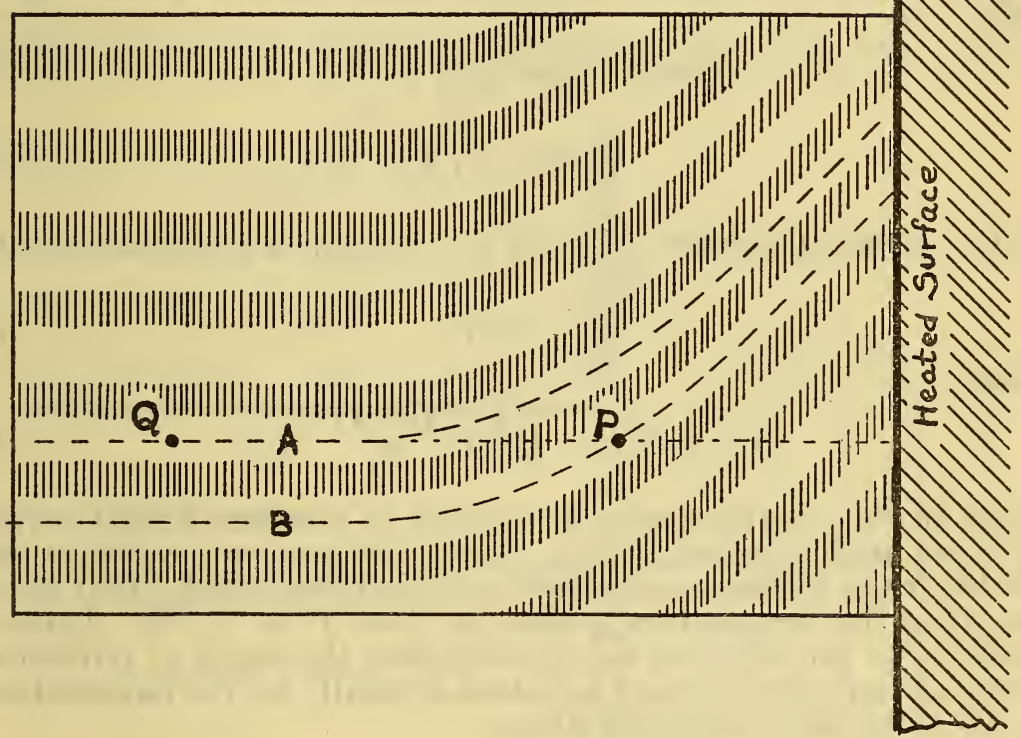

FigURe 2.-Method of measuring temperature from fringe shift

Since the index of refraction of air under the prevailing experimental conditions is of the order of 1.0003 the above equation reduces very closely to

$$
(n-1)=3 / 2 C \rho
$$

If, under one set of conditions, $N_{1}$ and $n_{1}$ are the number of light waves over a given path, and the index of refraction, respectively, and $N_{2}$ and $n_{2}$ are the same quantities under a second set of conditions then

$$
\begin{aligned}
& n_{1}=\frac{N_{1}}{N_{0}} \\
& n_{2}=\frac{N_{2}}{N_{0}}
\end{aligned}
$$

where $N_{0}$ is the number of light waves in the same path in a vacuum. If $L$ is the length of path under consideration, which is that of the

1 Lorentz, The Theory of Electrons, p. 145, 1909. 
heated surface $L_{0}$ plus a small end correction $\Delta L$, and if $\lambda_{0}$ is the wave length in vacuum of the light used then

$$
N_{0}=\frac{L}{\lambda_{0}}
$$

From equation (2) the change of index of refraction for a given change in density is

$$
n_{1}-n_{2}=3 / 2 C\left(\rho_{1}-\rho_{2}\right)
$$

Inserting the values given in equations (3) and (4) and rearranging yields

$$
\begin{aligned}
N_{1}-N_{2} & =3 / 2 C_{\rho_{1}} \frac{L}{\lambda_{0}}\left(1-\frac{\rho_{2}}{\rho_{1}}\right) \\
& =\left(n_{1}-1\right) \frac{L}{\lambda_{0}}\left(1-\frac{\rho_{2}}{\rho_{1}}\right)
\end{aligned}
$$

If the change in density is caused by a change in temperature only

whence

$$
\frac{\rho_{2}}{\rho_{1}}=T_{1} / T_{2}
$$

$$
N_{1}-N_{2}=\left(n_{1}-1\right) L / \lambda_{0} \frac{T_{2}-T_{1}}{T_{2}}
$$

Since in the interferometer the change in number of light waves in a given path is equal to the fringe displacement, equation (8) gives the fringe displacement as measured in fringe widths, that takes place when the temperature is changed from $T_{1}$ to $T_{2}{ }^{\circ} \mathrm{K}$. Letting $T_{1}$ and $n_{1}$ be the absolute temperature and the index of refraction or room air, equation (8) may be solved explicitly for the temperature rise above the air of the room giving

$$
T_{2}-T_{1}=T_{1} \frac{\Delta N}{\left(n_{1}-1\right) L / \lambda_{0}-\Delta N}
$$

The quantity $\left(n_{1}-1\right)$ is given in International Critical Tables ${ }^{15}$ for standard conditions and may be calculated for any conditions by using the gas laws, making proper correction for humidity. ${ }^{15}$

\section{END CORRECTION}

As previously mentioned the length $L=L_{0}+\Delta L$ includes the length of the heated surface $L_{0}$ plus a small correction $\Delta L$ which takes into account the fact that the layer of heated air extends beyond the ends of the hot surface. It was found that when no allowance was made for this end correction the temperature rise as calculated for the air at the surface of a $103 \mathrm{~mm}$ plate was greater by 5 per cent than the observed temperature rise of the plate itself.

If in equation (9) we insert for $T_{2}$ the observed temperature of the plate and for $\Delta N$ the observed fringe displacement at the plate surface, and solve for $L$, we find that $\Delta L=5 \mathrm{~mm} \pm 1 / 2 \mathrm{~mm}$. This value holds for all four cases under consideration as shown by the

18 I. C. T., vol. 7, pp. 4 and 5 . 
following values of $T_{2}-T_{1}$ at the plate surface as calculated from the observed fringe displacement at the plate.

\begin{tabular}{|c|c|}
\hline \multicolumn{2}{|c|}{$\left(T_{2}-T_{1}\right)$} \\
\hline Observed & Calculated \\
\hline & \\
\hline$\circ C$. & $\circ C$. \\
118.1 & 118.9 \\
63.5 & 63.8 \\
29.4 & 29.4 \\
14.3 & 14.3 \\
\hline
\end{tabular}

This method of calculating $\Delta L$ at the surface of the plate neglects any discontinuity of temperature between the plate and the air at its

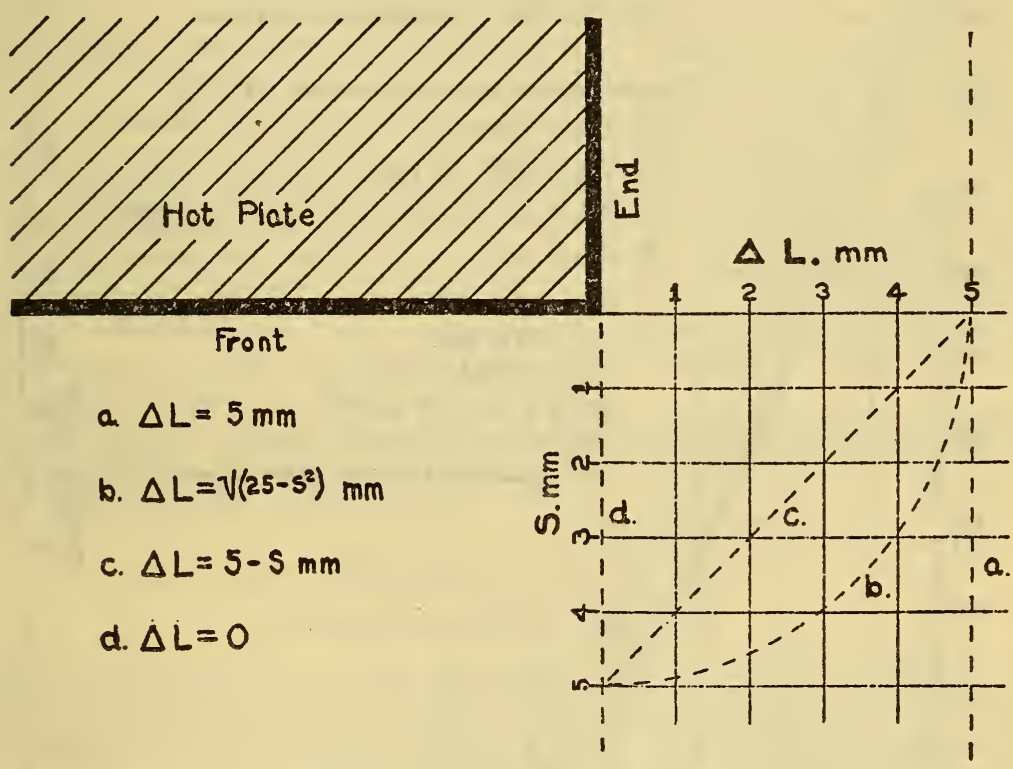

Figure 3.-Variation of the end correction with distance from the plate on several assumptions

surface. Although the kinetic theory indicates the existence of such a discontinuity it is so small that it can be entirely neglected under the conditions of these experiments.

Having determined the value of $\Delta L$ at the surface of the plate there remains the problem of determining its approximate value at any point in the region of heated air. As the quantity involved is small, a purely empirical solution was considered sufficient. As one limit (fig. 3 ) let us assume (a) that $\Delta L=5 \mathrm{~mm}$ remains constant throughout this region. For another limit let us assume (c) that $\Delta L$ decreases linearly to zero at some point, say for convenience $5 \mathrm{~mm}$ in front of the plate. Between these two limits a circle $(b)$ has been arbitrarily drawn. For any given distance in front of the plate, $\Delta L$ was chosen as the corresponding semichord of this circle in the final computation of the results. 
In Figure 4 is shown the average displacement of the center line of the interference fringes for a total temperature rise of $118.1^{\circ} \mathrm{C}$. as measured from the photograph in Figure 5. The scale for $\Delta N$ in fringe widths is shown on the right and that for temperature rise on the left. These scales have been so chosen that the fringe displacement at the plate coincides with the temperature rise at the same point.

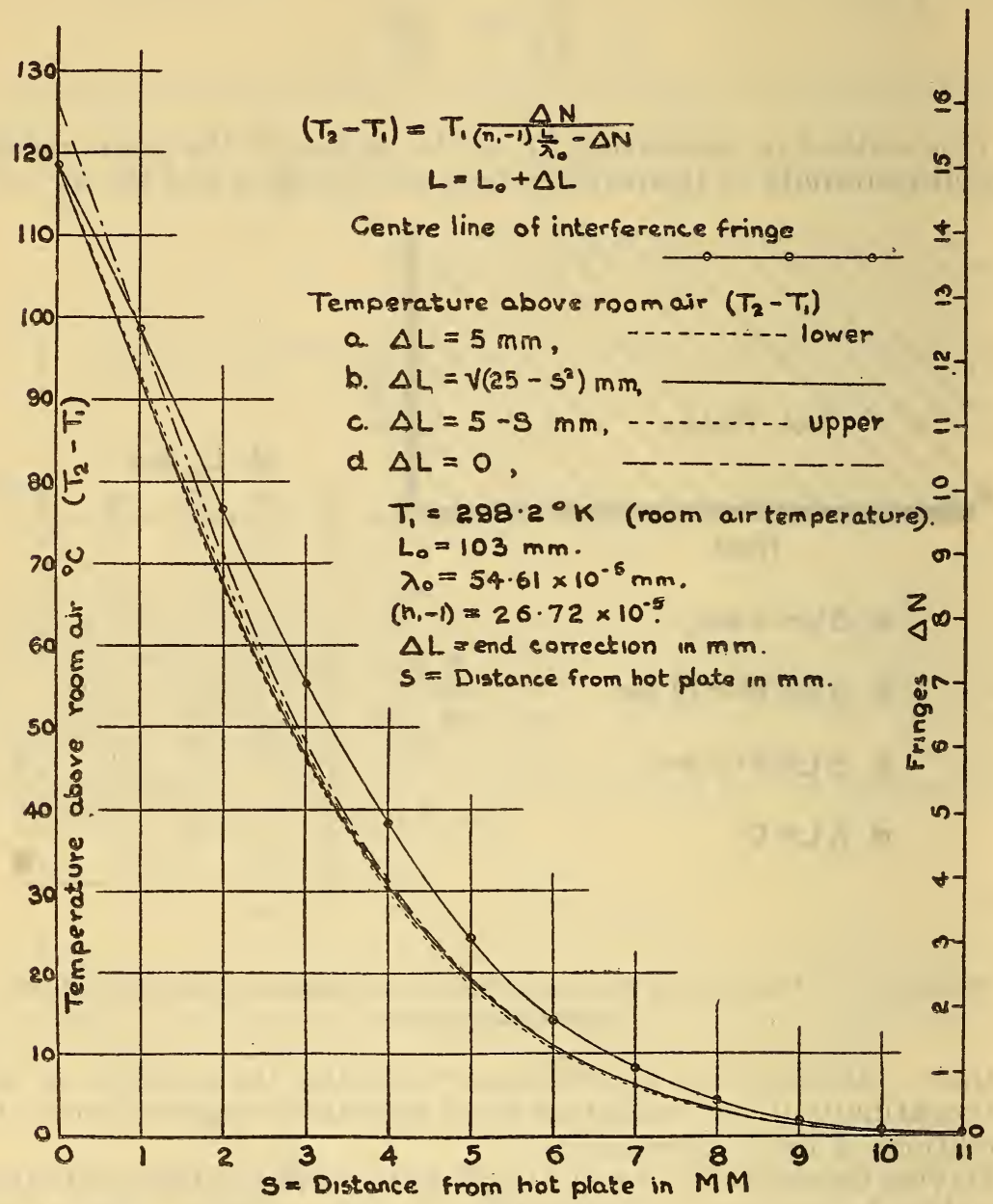

FIGURE 4.-Variation of fringe shift and of temperature with distance from a hot plate, the latter corrected for end effect according to several assumptions

Below the observed fringe displacement, four curves are shown which give the corresponding temperatures as calculated for four different assumptions for the end correction. This figure shows clearly the justification of the empirical choice of $\triangle L=\sqrt{25-S^{2}}$ as the end correction.

It is clear from this figure, as well as from equations (8) and (9) that the interference fringe does not represent the temperature line. 
The difference, however, becomes much less as the total temperature rise becomes smaller.

\section{EFFECTS OF ABERRATION}

Another factor that needs to be considered is that not every ray through a given point in the focal plane has come over the same path in front of the hot surface. Some of the rays may have come through warmer, and some through colder regions of air. At a distance from the plate these effects are likely to balance each other and no appreciable error results. Furthermore, the effect can be reduced by using a small aperture in front of the camera lens. If a 3 $\mathrm{mm}$ aperture is used, with the focus set on one edge of the plate, the cone of light entering the lens from a point in the focal plane will have a diameter at the other edge of $0.55 \mathrm{~mm}$.

At the surface of the plate the error will be greatest, and it may be shown that it will amount to the difference between the temperature of the plate and that at a distance of $0.12 \mathrm{~mm}$ on the assumption that all rays within the half cone are equally probable and that an average is taken of each ray over its full length across the plate. The above value also assumes perfect collimation of the set.

As even with the greatest care, errors due to collimation will occur, an aperture of $6 \mathrm{~mm}$ was used in the majority of the exposures. By this increase in aperture the time of exposure could be reduced to 6 seconds. With the $3 \mathrm{~mm}$ aperture an exposure of more than 20 seconds was required and many plates were spoiled by stray air currents which caused the fringes to move.

It will be noted that the purely empirical method used in making the end correction tends also to correct for small errors caused by aberration and imperfect collimation.

\section{VERTICAL HOT PLATE}

\section{EXPERIMENTAL RESULTS}

Four photographs showing the fringe displacement in front of a vertical hot plate are shown in Figure 5. In each photograph the edge of the plate is seen at the extreme right. The straight part of the fringes at the left indicates room air temperatures. The hot plate is $210 \mathrm{~mm}$ high and $103 \mathrm{~mm}$ wide. In each case the center of the plate is shown at the bottom of the photograph. The temperature difference between room air and the hot plate is given with each figure. The room air temperature varied from $21.7^{\circ}$ to $25.2^{\circ} \mathrm{C}$., and was measured by a shielded thermocouple $T$ shown in Figure 1. The plate temperature was measured by a thermocouple soldered to one edge of the plate. The photograph of the millimeter scale was obtained by a double exposure on one of the photographs of the fringes, all of which are enlarged to the same scale.

Figure 6 shows the effect of the total temperature rise on the relative temperature distribution. In the preliminary report ${ }^{16}$ on this work, it was stated that the distribution was the same for all temperatures, any differences which occurred being ascribed to experimental error. Repeated measurements have since been made on the enlarged photo-

${ }^{16}$ Kennard, Phys. Rev., vol. 38, p. 1921, 1931.

$117348-32-9$ 
graphs which indicate that there is a small but systematic effect as shown in the figure. In the terms of the film theory, the straight portions of the curves near the plate surface indicate that the temperature coefficient of film thickness is approximately -0.12 per cent per degree. Lines $a^{\prime}, d^{\prime}$, and $d^{\prime \prime}$ will be discussed later.

\section{DISCUSSION OF FIGURE 6}

No function has as yet been developed which represents the observed temperature distribution in front of a vertical hot plate. It

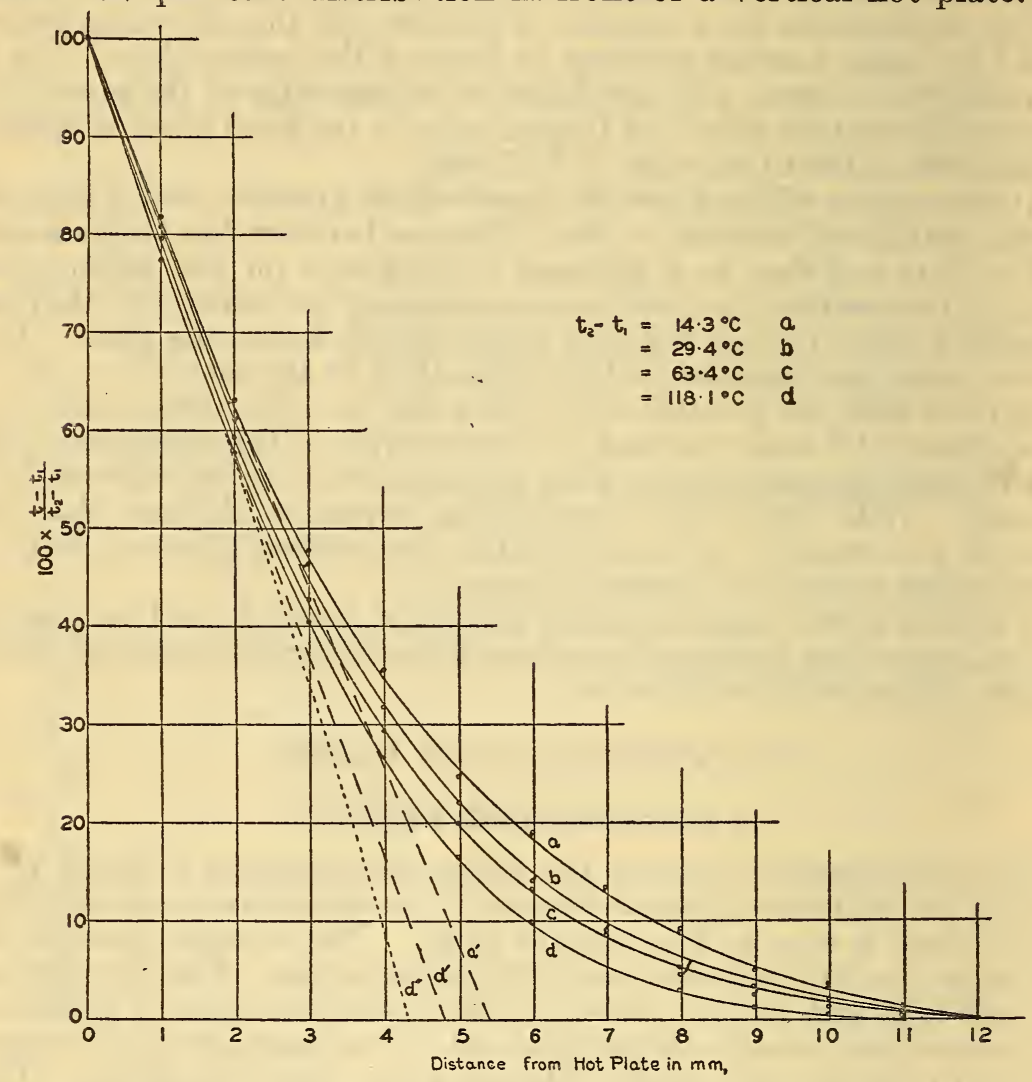

FIGURE 6.-Effect of total temperature rise on relative temperature distribution for vertical plate

will be noted that the curves in Figure 5 show no sharp change in slope or in curvature, and that the curves approach the room temperature line asymptotically. Whatever the above function may be, it seems evident from these curves that it is continuous both in its first and second derivatives.

These curves may be interpreted as follows: Near the surface the warm air rises parallel to the plate with very nearly stream line or laminar flow. In this region which includes 40 or 50 per cent of the 
temperature drop the heat transfer closely approximates pure conduction. Cold air from a distance is drawn into this rising column of warm air. As the cold air approaches the plate it is heated either by pure conduction or by fine grained turbulence. The presence of large scale vortex turbulence is ruled out by the fact that the interference fringes remain sharp throughout the field over a six-second exposure. Fine grained turbulence, in which the vortices have a diameter of $1 / 10$ $\mathrm{mm}$ or less, may exist and might account for the shape of the curves for the lower half of the temperature drop by the mixing of the warmer and colder air.

For the middle portion of a short plate as used in this experiment, the observed temperature distribution may be accounted for without the assumption of turbulence. For a long plate, at points far from the bottom the assumption of turbulence seems necessary to account for the fact that the convective heat transfer is practically independent of the height.

\section{CALCULATION OF CONVECTIVE HEAT TRANSFER}

From the temperature gradient at the surface of the plate, assuming pure conduction in this region one may calculate the rate of convective heat transfer per unit area.

$$
Q=-K \frac{d T}{d s}
$$

where $K$ is the conductivity of the air taken at the plate temperature.

For a temperature rise of $118.1^{\circ} \mathrm{C}$. with the plate at $143.3^{\circ} \mathrm{C}$.

and

$$
K=0.000308 \text { watt } \mathrm{cm}^{-1}{ }^{\circ} \mathrm{C}^{-1}
$$

whence

$$
\frac{d T}{d s}=246^{\circ} \mathrm{C} \cdot \mathrm{cm}^{-1}
$$

$$
Q=0.0760 \text { watt } \mathrm{cm}^{-2}
$$

For a temperature rise of $14.3^{\circ} \mathrm{C}$. with the plate at $36.8^{\circ} \mathrm{C}$.

and

$$
K=0.000246 \text { watt } \mathrm{cm}^{-1}{ }^{\circ} \mathrm{C} .{ }^{-1}
$$

whence

$$
\frac{d T}{d s}=27^{\circ} \mathrm{C} \cdot \mathrm{cm}^{-1}
$$

$$
Q=0.00666 \text { watt } \mathrm{cm}^{-2}
$$

If we set $\frac{Q_{1}}{Q_{2}}=\left(\frac{\Delta t_{1}}{\Delta t_{2}}\right)^{n}$ we find $n=1.17$ which is considerably less than the value 1.25 generally taken, but agrees very well with the value found by Griffith and Davis for short vertical cylinders. Their value, however, is for the total heat emitted, whereas the above value applies to the particular portion of the plate photographed. 


\section{HORIZONTAL CYLINDERS}

\section{EXPERIMENTAL RESULTS}

Photographs were taken of the interference fringes about horizontal heated cylinders. Figure 7 shows a composite photograph about a cylinder $3 \mathrm{~cm}$ diameter and $10 \mathrm{~cm}$ long. The cylinder is made of two coaxial brass tubes with the heating coil between. The supports are vertical transite plates $3 \mathrm{~mm}$ thick which are inserted into the ends of the inner cylinder and extend $1 \frac{11}{2} \mathrm{~cm}$ beyond the end. A thermocouple, soldered to the back of the outer cylinder gives the surface temperature.

The photographs were taken in the order upper left, lower left, bottom, and top. The imperfect match between the top and the upper left photograph can be accounted for by a slight shift in the adjustment of the interferometer in the three hours necessary to complete the set. Such a change has no effect in plotting the temperature field as each photograph is taken as a unit.

In Figure 8 the isothermal lines have been drawn about the cylinder by connecting the points of equal fringe displacement. The correction for end effect was made by assuming that the fringe displacement at the surface corresponds to air having the temperature of the surface of the cylinder, with proportional correction for the lower temperatures. The outer line indicates the boundary of the region of heated air. Along this line the temperature is less than $0.3^{\circ} \mathrm{C}$. above that of the room. From the fringes in Figure 7 it will be seen that the departure from room temperature is at first very gradual. The exact position of the outside boundary is therefore indefinite.

One may safely assume that at the surface of the cylinder the air flow is approximately laminar and that the heat is transferred by pure conduction. Under such conditions the heat flux will be directly proportional to the temperature gradient, which can be determined from the spacing of the isothermal lines. If we take the heat flux at the bottom as 100, we find from Figure 9 that it decreases uniformly to 70 at the side. This uniform decrease continues to about $45^{\circ}$ from the top where the heat flux begins to drop off more rapidly, so that at the top it is only 10. However, the isothermals shown at the top of the cylinder are so far from the surface that it is not safe to assume laminar flow throughout this region. In case of turbulence the heat flux might be considerably greater than that indicated by the average temperature gradient over the distances involved.

In Figure $9 A$ is shown a composite photograph of the fringes about a cylinder $1.27 \mathrm{~cm}$ diameter and $10 \mathrm{~cm}$ long. The cylinder is of brass tubing, having a $1 \mathrm{~mm}$ wall and is lined with asbestos paper which is held in place by the heating coil. The leads to the heating coil project a few millimeters beyond the cylinder and are then bent sharply to the right and serve as the support. The cylinder is open at both ends and the interference fringes are clearly seen through the hollow center. The surface temperature of the cylinder is determined by the thermocouple wires shown in the lower right of the photograph.

In Figure $10(A)$ are shown the isothermal lines about the above cylinder. They were determined as in Figure 8 by plotting the points of equal fringe shift. From the spacing of the isothermal lines 


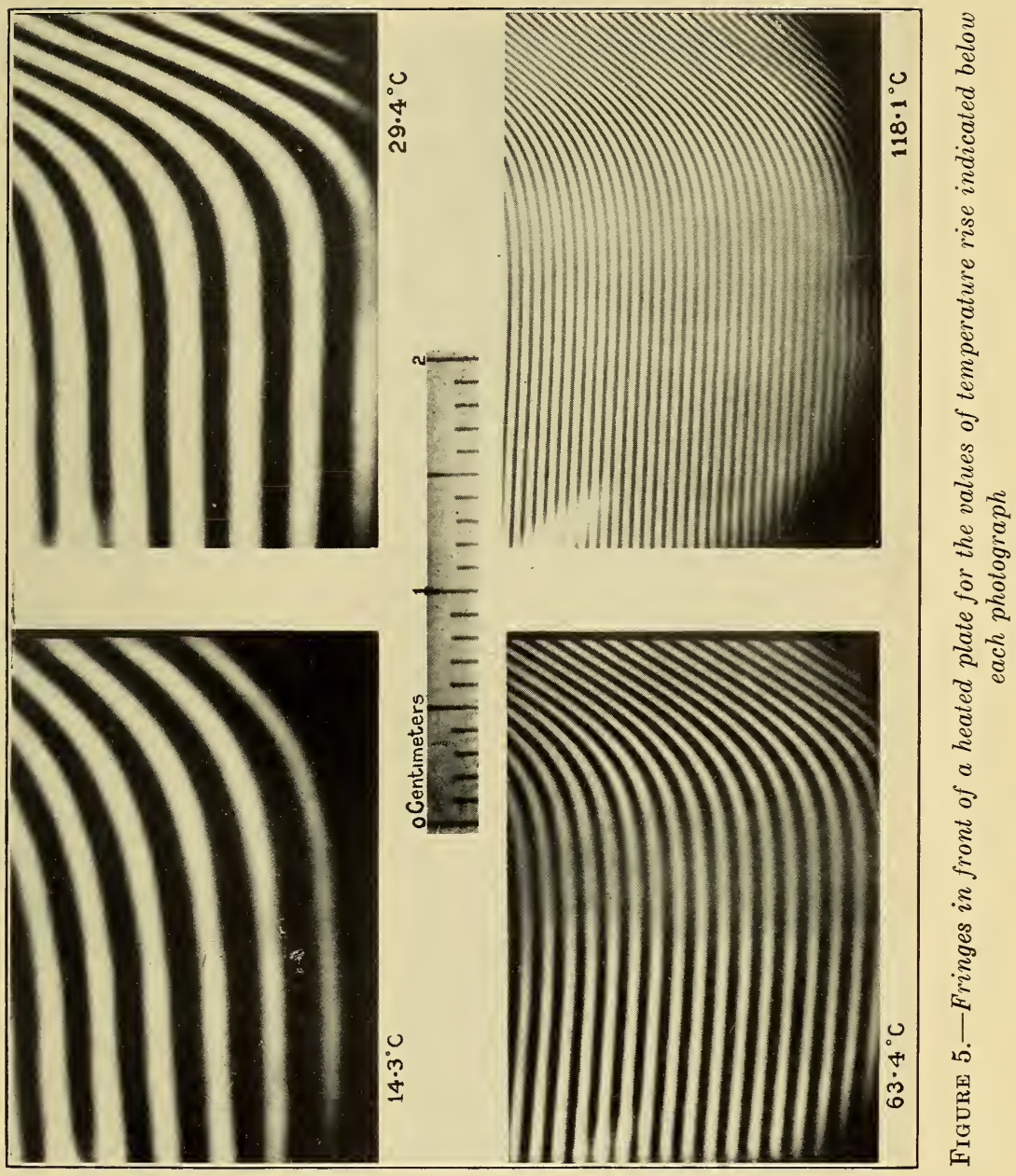


B. S. Journal of Research, RP452

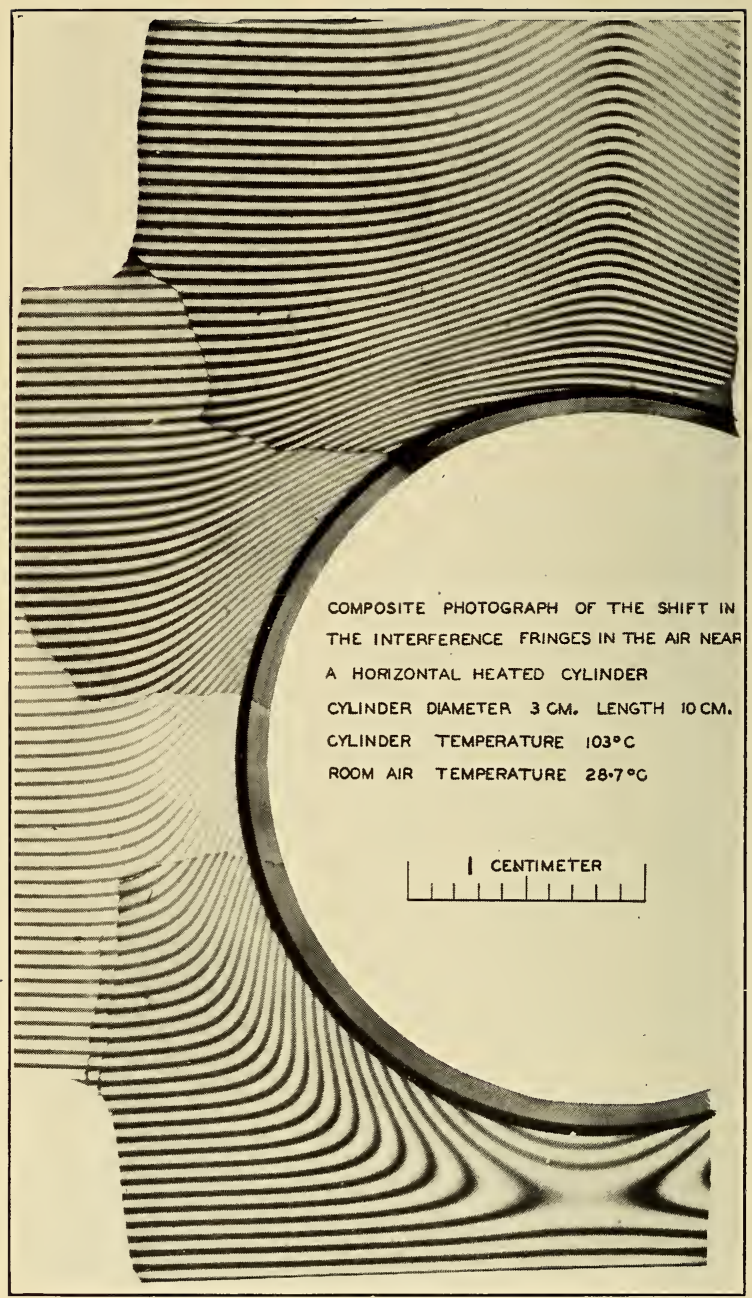

Figure 7 
near the cylinder the heat flux is found to decrease from 100 at the bottom, to 80 at the side, and to 20 at the top.

Figure $9(B)$ shows a photograph of the fringes about a cylinder $5 \mathrm{~mm}$ in diameter and $10 \mathrm{~cm}$ long. The walls are $1 \mathrm{~mm}$ thick and lined with alundum cement. A straight nichrome wire serves as both heating element and support. The thermocouple giving the surface temperature is shown at the right center with the heating and support

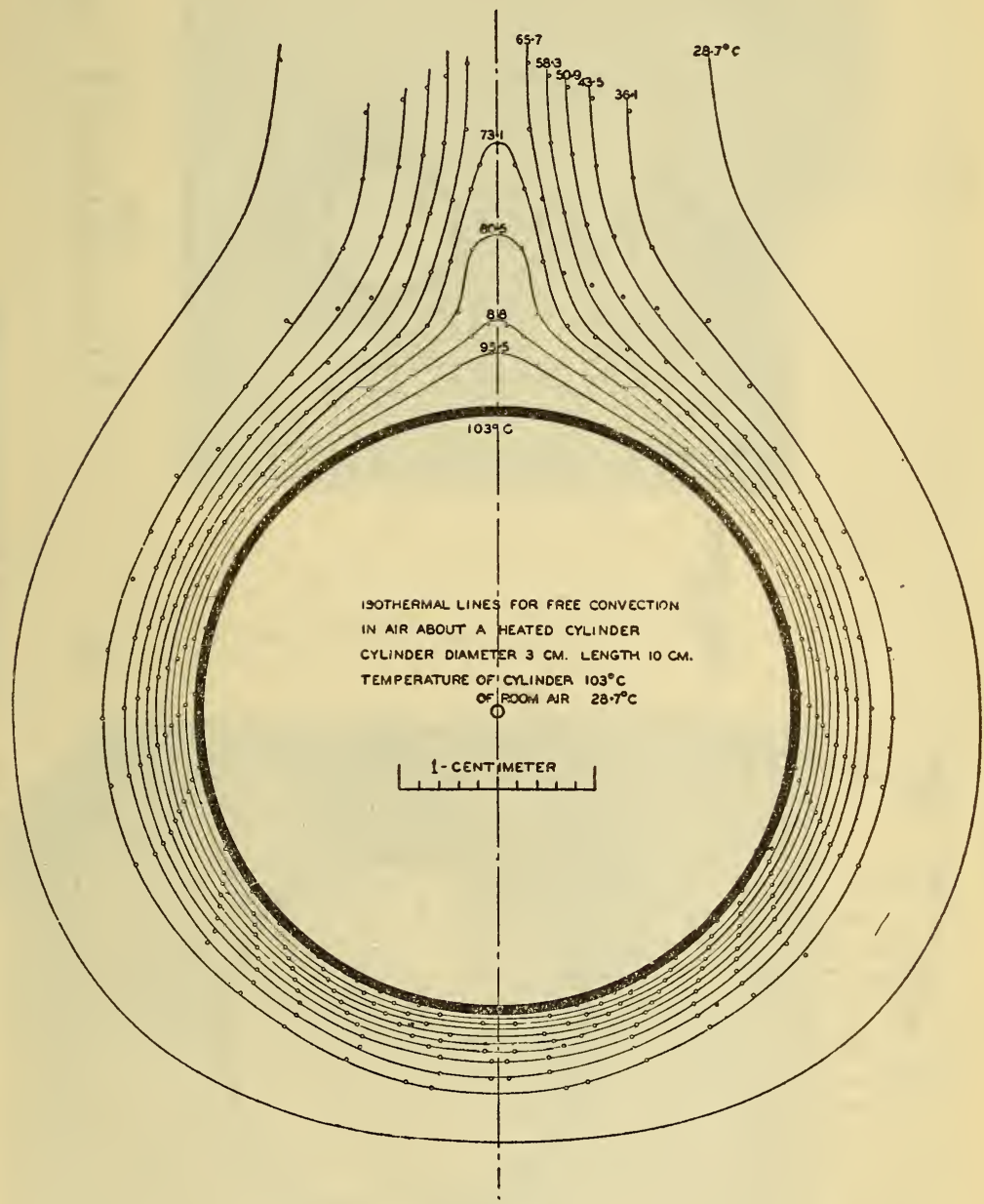

FIGURE 8

wire below. Interference fringes may be seen through the hollow center of the tube. As the hole is $100 \mathrm{~mm}$ long and only about $1 \mathrm{~mm}$ in diameter, the appearance of these fringes gives an idea of the collimation of the apparatus.

The isothermal lines for this cylinder are shown in Figure $10(B)$. From the spacing of the isothermals the heat flux is found to decrease from 100 at the bottom to approximately 90 at the side, and to 30 at the top. 


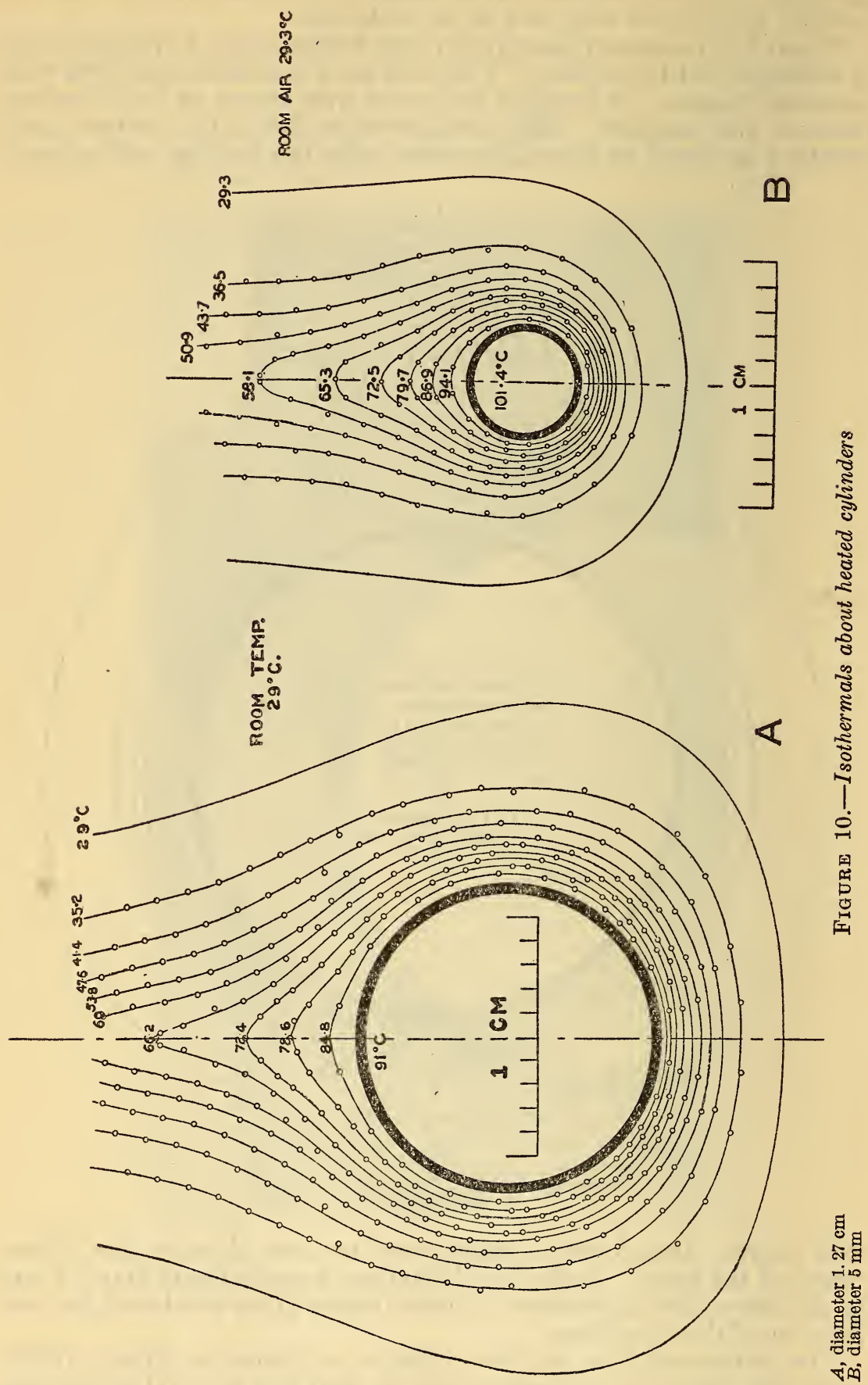




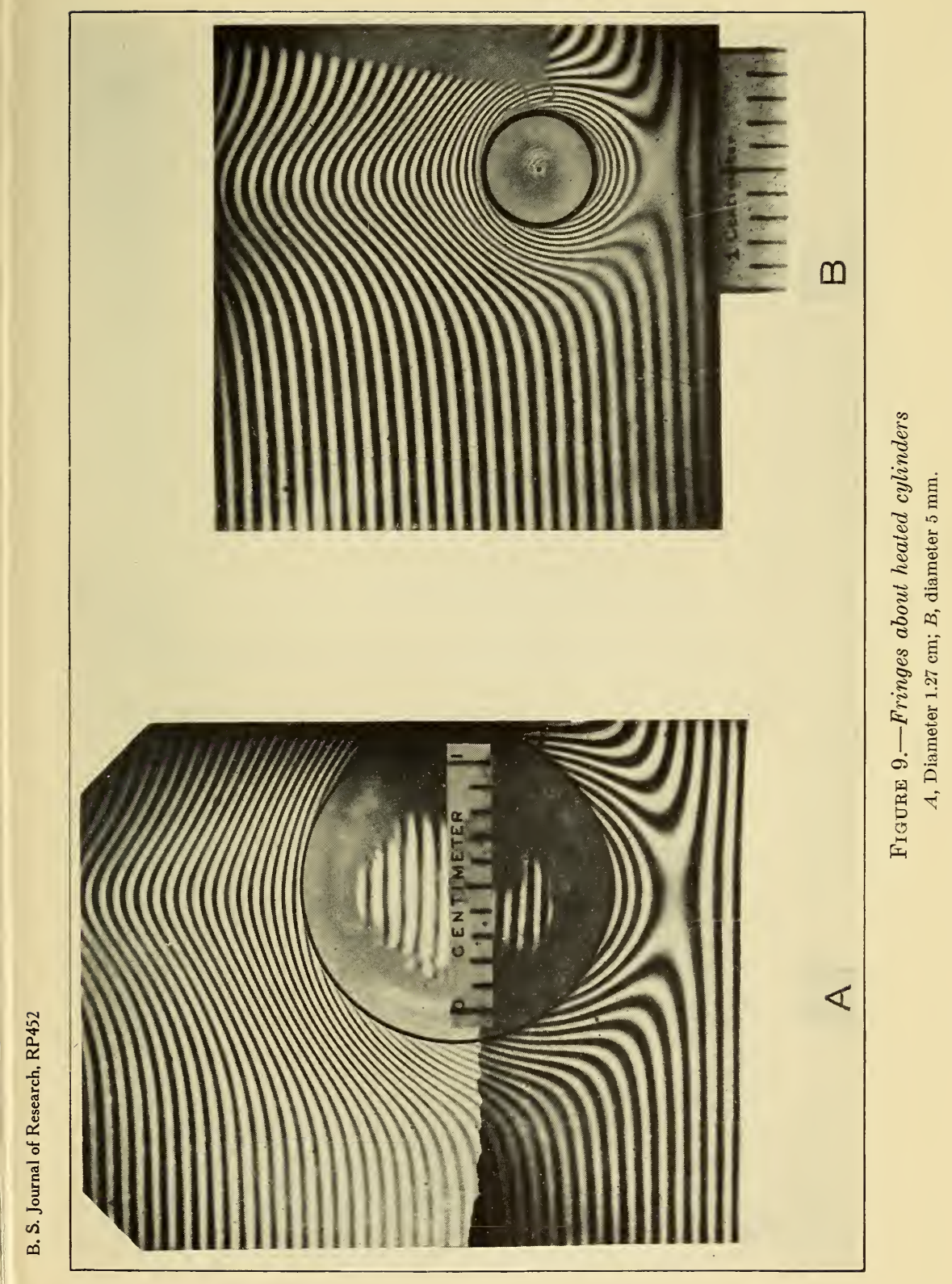


B. S. Journal of Research, RP452

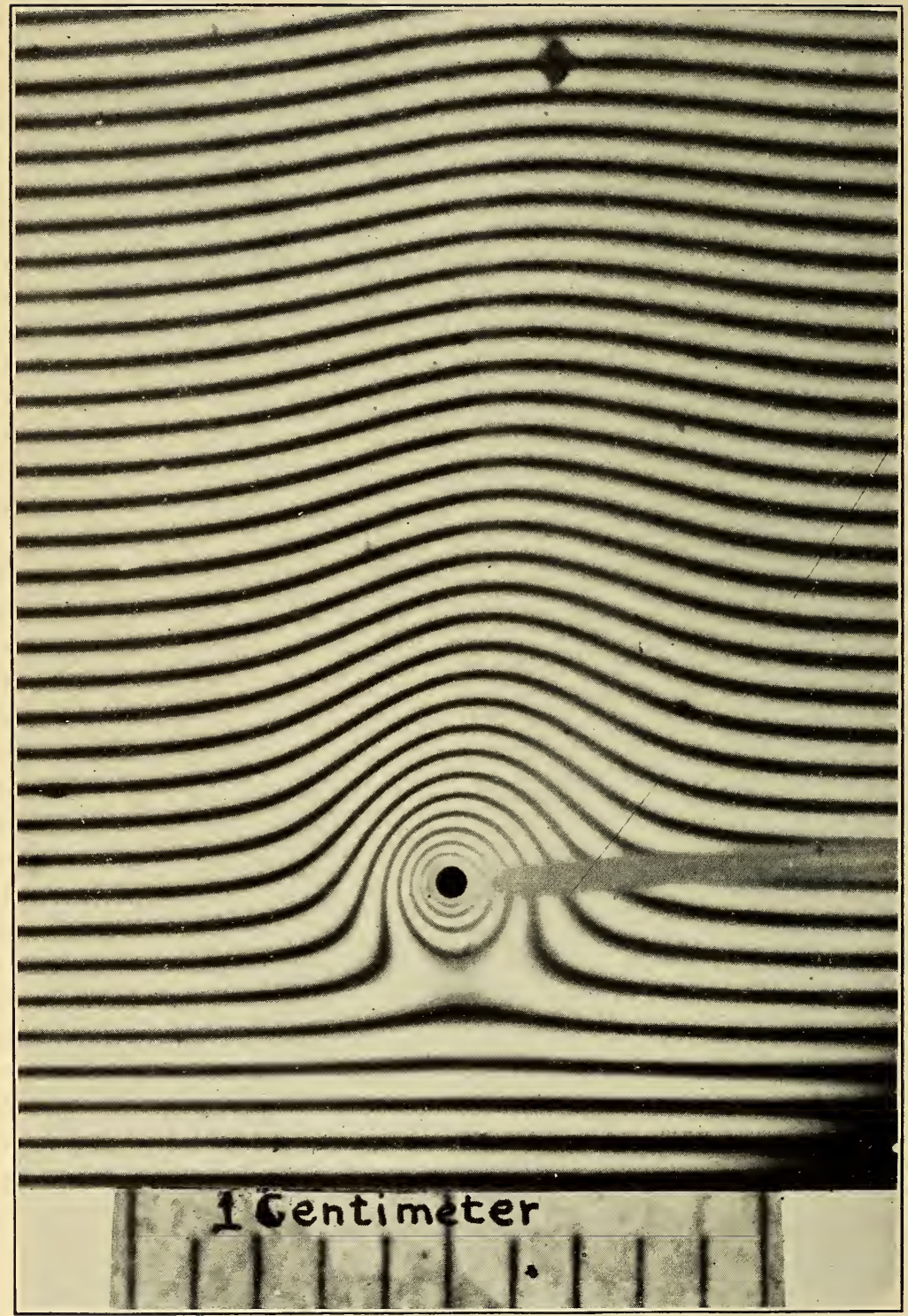

Figure 11.-Fringes about a wire $0.4 \mathrm{~mm}$ in diameter 
In Figure 11 is shown a photograph of the fringes about a horizontal platinum wire $0.4 \mathrm{~mm}$ in diameter. The current and the potential drop were measured on a potentiometer and the temperature determined from the resistance. Potential leads were used so that the resistance measured was only that of the straight portion of the wire about which the fringes were photographed and did not include the leads. In order to throw the leads out of focus and to reduce the aberration error the fringes were brought to a focus at the middle of the wire.

The isothermal lines about this wire are shown in Figure 12. It was not possible to get as many points of equal fringe displacement as

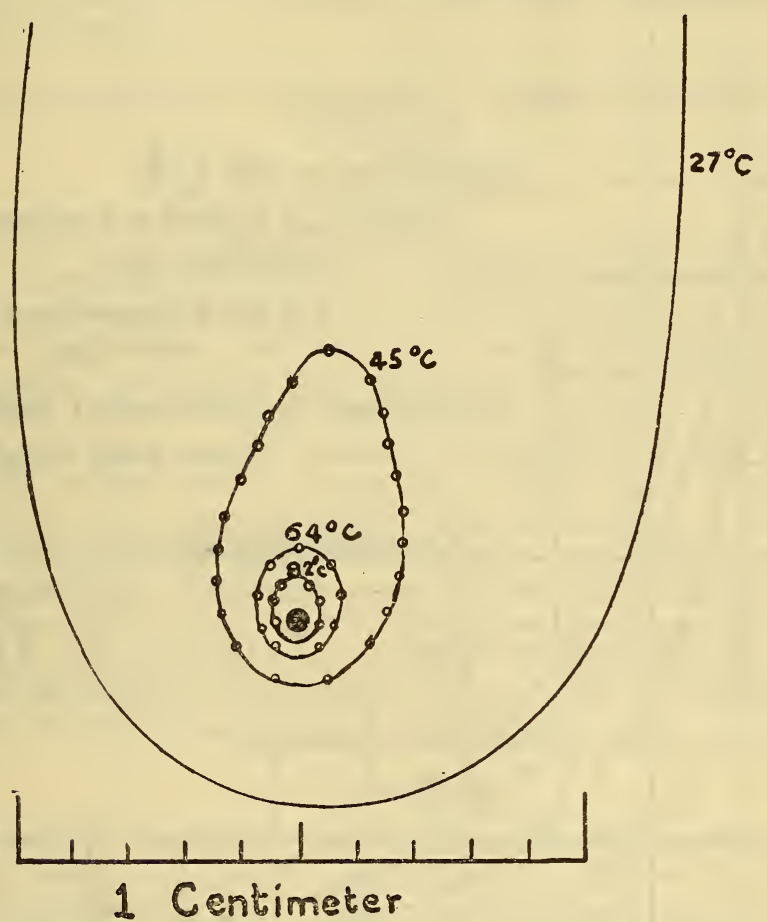

FIGURE 12.-I sothermals about a wire $0.4 \mathrm{~mm}$ in diameter

in the case of the other cylinders, and only four isothermals have been drawn. These are sufficient, however, to show that the heat flux changes approximately from 100 at the bottom to 90 at the side, and to 30 at the top.

Figure 13 gives the temperature distribution for the four cylinders in a horizontal plane containing the cylinder axis. The curves for the distribution in a vertical plane below the cylinders are similar to those in this figure but closer to the surface. It will be noted that the curves in Figure 13 show no evidence of discontinuity in slope or curvature. 


\section{DISCUSSION}

The temperature distribution for radial heat conduction from a cylinder for constant heat flow may be expressed mathematically by

$$
t=A-B \log r
$$

where $t$ is the temperature and $r$ the distance from the center.

The constants $A$ and $B$ may be determined by inserting the observed values of $t$ and $r$ at the surface of the cylinder and at some other

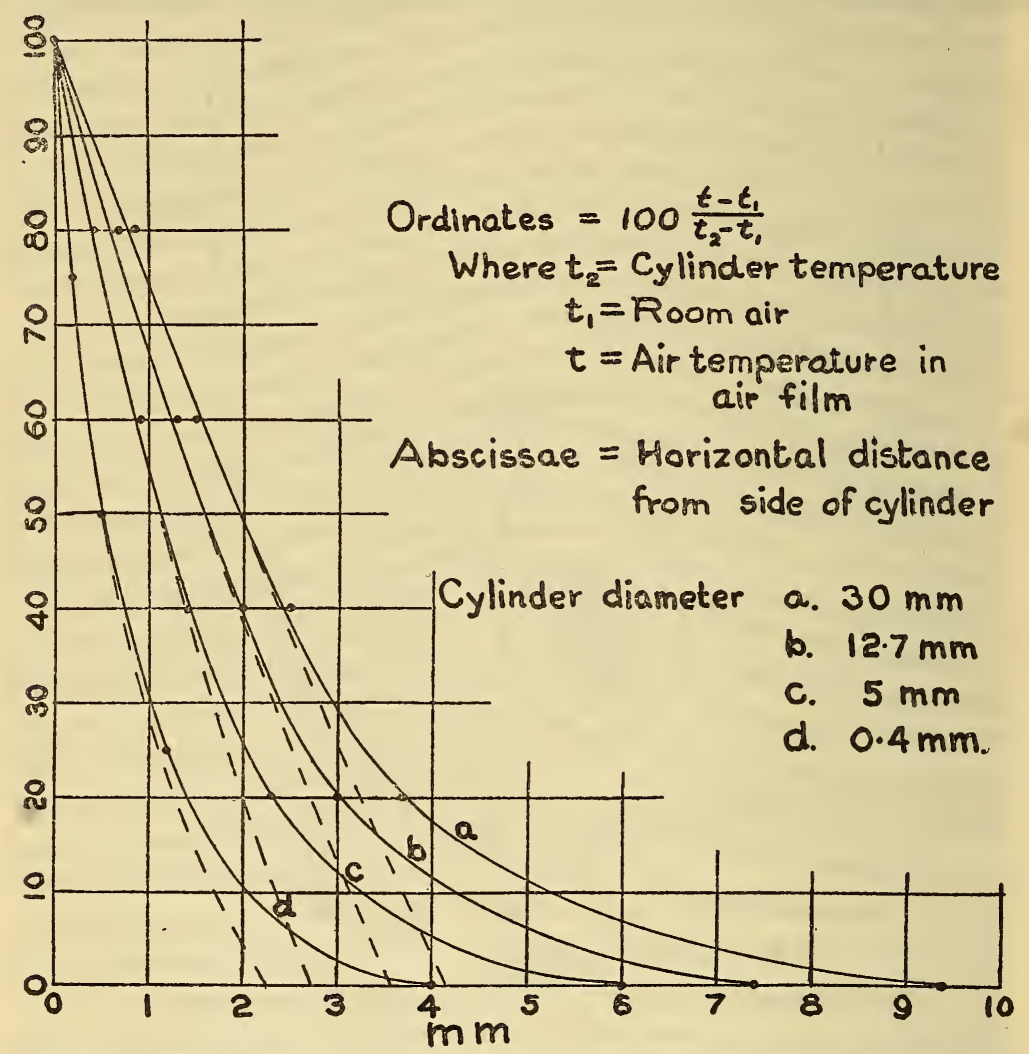

FIGURE 13.-Relative temperature distribution for cylinders as measured experimentally compared with the film theory

point for which $t$ and $r$ are known. If we take for $t$ and $r$ the room air temperature and the horizontal radius to the point at which this temperature is reached, the values for $A$ and $B$ so obtained will give a temperature distribution for pure conduction that corresponds only at the end points with that observed. If, however, the observed point for half the temperature drop is chosen, it is found that the temperature distribution as calculated for pure conduction coincides closely with that obtained from the fringe shift for the upper half of the temperature drop. For the lower half of the temperature drop there is a wide divergence. The broken lines in Figure 13 represent 
the temperature distribution for pure conduction when fitted to the point of mean temperature drop as indicated above.

The curves in Figure 13 may be interpreted as were those for the vertical hot plate in Figure 6 . That is, at the bottom and the sides of the cylinder, heat is transferred for the first half of the temperature drop under conditions closely approximating pure conduction. The temperature gradient then becomes much less than that called for by radial conduction. The change in temperature gradient may be accounted for by the presence of fine-grained turbulence, or by assuming that cold air is drawn in with laminar flow and heated.

No interpretation is offered of the temperature distribution at the top of these cylinders, as conditions with regard to turbulence in this region are not known.

\section{COMPARISON OF OBSERVED RESULTS WITH FILM THEORY}

\section{VERTICAL PLATE}

It is of interest to contrast the temperature distribution as found from these experiments with that assumed by the film theory. In the case of the vertical hot plate the curve representing temperature distribution under the conditions assumed in the film theory would be very nearly a straight line, ending at room temperature at 4.3 $\mathrm{mm}$ from the plate. ${ }^{17}$ This curve would be slightly convex upward because of the decrease in conductivity of the air with decrease in temperature, which would cause an increase in the temperature gradient at the lower temperatures. The dotted line $d^{\prime \prime}$ in Figure 5 shows the temperature distribution under the conditions assumed in the film theory, taking into consideration the change of thermal conductivity with temperature. The broken lines $a^{\prime}$ and $d^{\prime}$ are straight and indicate the temperature gradient at the surface. The wide departure of the observed temperature distribution from that assumed by the film theory is clearly seen in this figure.

\section{CYLINDERS}

A sketch of the conditions assumed to exist for heat transfer from cylinders as given by Rice ${ }^{18}$ is reproduced in Figure 14 . The temperature distribution under these assumptions will have radial symmetry and will, along any radius, be represented by curves similar to the broken lines in Figure 14. From the isothermal lines shown in Figures $8,10(A), 10(B)$, and 12 it is certain that radial symmetry does not exist. There is also no evidence that the temperature distribution in any radial plane corresponds to the conditions of pure conduction through a film of limited thickness. At the bottom and sides of the cylinder the temperature distribution does, however, correspond to pure conduction for the upper half of the temperature drop.

It will be seen that the conditions implied by the film theory do not hold for free convection in air from a vertical hot plate nor from horizontal heated cylinders from $0.4 \mathrm{~mm}$ to $30 \mathrm{~mm}$ in diameter. It is not likely that these assumptions hold more closely under other 
conditions. Drew, Hogan, and McAdams ${ }^{19}$ have shown the complete lack of radial symmetry of the heat flux in the case of forced convection in air from a pipe normal to the air stream. Griffith and Davis ${ }^{20}$ show, for free convection from a vertical hot plate, that there is a variation of the temperature distribution with distance from the bottom of the plate. This variation is much more marked
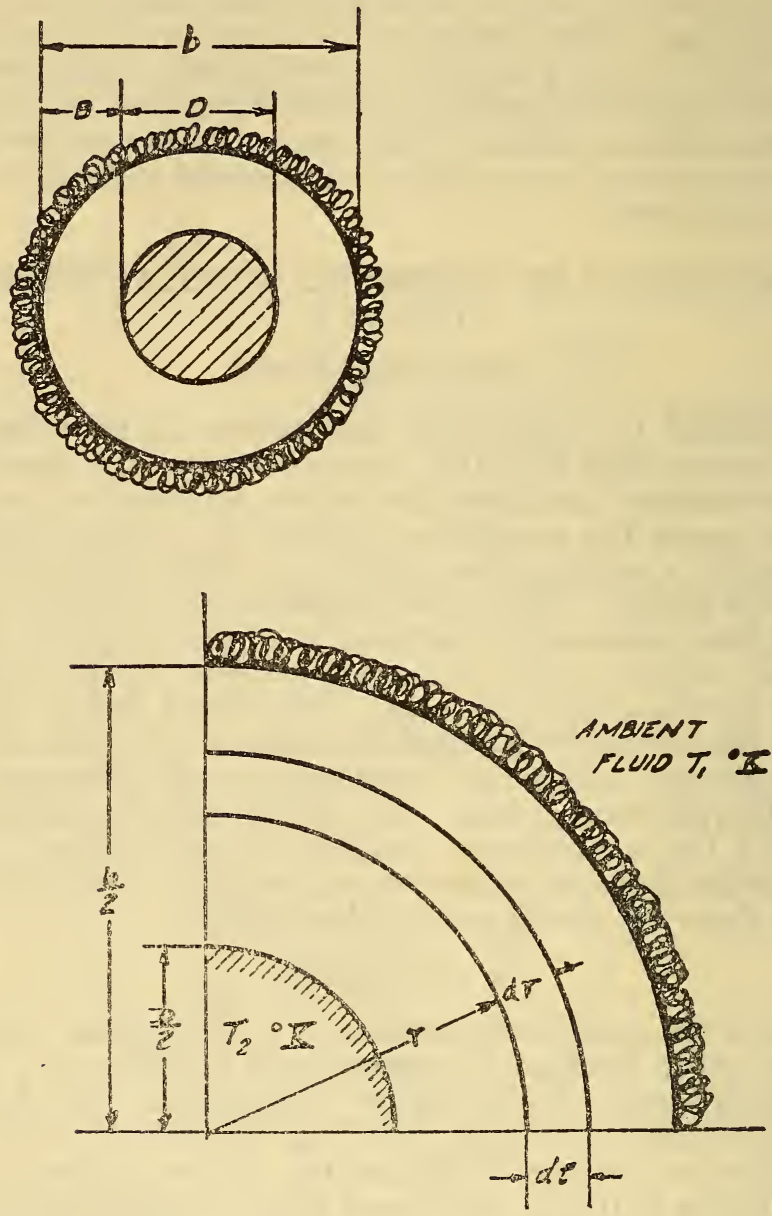

FIGURE 14.-Film theory for cylinder, according to Rice

(Reproduced from Trans. A. I. E. E., vol 42, p. 653, 1923.)

for forced convection from a hot plate as shown by Jürges ${ }^{21}$ and Elias ${ }^{22}$ The film theory permits no such variation.

As actually used ${ }^{23}$ to calculate convective heat transfer, the film theory serves merely as a device by which experimental results may be correlated by means of dimensional analysis. Experimentally determined coefficients of heat transfer could serve the purpose equally

19 Ind. Eng. Chem., vol. 23, p. 936, 1931.

20 See footnote 10, p. 789 .

21 See footnote 11, p. 789 .

${ }^{22}$ See footnote 13, p. 789

23 I. C. T., vol. 5, p. 234. 
well, and not be open to the criticism of being based on a false physical assumption. The assumption of a film or any other device is not necessary for this purpose as indicated by the work of Fishenden and Saunders. ${ }^{24}$

\section{CONCLUSION}

An apparatus has been devised by which the temperature of the air near heated surfaces may be determined without the use of any material thermometric device. This is accomplished by measuring the displacement of the fringes in a suitable interferometer. A series of photographs have been taken of the interference fringes near a heated vertical plate and about heated horizontal cylinders of different diameters. The results show that the temperature distribution does not conform to that assumed by the film theory.

The apparatus also makes it possible to determine the temperature gradient in the air at any point on the heated surface, thus making it possible to calculate the heat flux at that point. This has not been possible with any apparatus heretofore developed.

\section{ACKNOWLEDGMENT}

The writer wishes to acknowledge his indebtedness to H. C. Dickinson, who suggested the possibility of using the technic of interferometery to study the conditions in the fluid near a solid heated surface. He wishes also to thank M. S. Van Dusen for valuable suggestions in the interpretation of results.

The writer also wishes to make special acknowledgment of his indebtedness to the Johns-Manville Corporation, who made possible this investigation by the establishment at the Bureau of Standards of the L. B. McMillan Fellowship for fundamental studies in the transfer of heat between solids and fluids.

Noте.-After the manuscript of this paper was completed, the paper by Hansen (Zeit für Tech. Phys. XII, 9, 1931) was received. Hansen describes the design and construction of an elaborate interferometer for use in work similar to that described here, and goes into the optical theory in detail. As concerns heat transfer he does not go beyond showing that it is possible to obtain photographs of the interference fringes displaced by a heated cylinder.

Washington, April 22, 1932.

"See footnote 6, p. 788. 